\title{
Dual Mode Sensing with Low-Profile Piezoelectric Thin Wafer Sensors for Steel Bridge Crack Detection and Diagnosis
}

\author{
Lingyu Yu, ${ }^{1}$ Sepandarmaz Momeni, ${ }^{2}$ Valery Godinez, ${ }^{2}$ \\ Victor Giurgiutiu, ${ }^{1}$ Paul Ziehl, ${ }^{3}$ and Jianguo $\mathbf{Y u}^{3}$ \\ ${ }^{1}$ Department of Mechanical Engineering, University of South Carolina, Columbia, SC 29208, USA \\ ${ }^{2}$ Mistras Group Inc., 195 Clarksville Road, Princeton Junction, NJ 08550, USA \\ ${ }^{3}$ Department of Civil Engineering, University of South Carolina, Columbia, SC 29208, USA
}

Correspondence should be addressed to Lingyu Yu, yu3@cec.sc.edu

Received 30 May 2011; Accepted 6 September 2011

Academic Editor: Piervincenzo Rizzo

Copyright (c) 2012 Lingyu Yu et al. This is an open access article distributed under the Creative Commons Attribution License, which permits unrestricted use, distribution, and reproduction in any medium, provided the original work is properly cited.

\begin{abstract}
Monitoring of fatigue cracking in steel bridges is of high interest to many bridge owners and agencies. Due to the variety of deterioration sources and locations of bridge defects, there is currently no single method that can detect and address the potential sources globally. In this paper, we presented a dual mode sensing methodology integrating acoustic emission and ultrasonic wave inspection based on the use of low-profile piezoelectric wafer active sensors (PWAS). After introducing the research background and piezoelectric sensing principles, PWAS crack detection in passive acoustic emission mode is first presented. Their acoustic emission detection capability has been validated through both static and compact tension fatigue tests. With the use of coaxial cable wiring, PWAS AE signal quality has been improved. The active ultrasonic inspection is conducted by the damage index and wave imaging approach. The results in the paper show that such an integration of passive acoustic emission detection with active ultrasonic sensing is a technological leap forward from the current practice of periodic and subjective visual inspection and bridge management based primarily on history of past performance.
\end{abstract}

\section{Introduction}

According to the Federal Highway Administration (FHWA) National Bridge Inventory (NBI) of 2007, the number of structurally deficient and functionally obsolete bridges is 72,524 and 79,792 , respectively [1]. While there are about 10,000 bridges being constructed, replaced, or rehabilitated annually in the United States at a cost of over $\$ 5$ billion, the total annual costs including maintenance and routine operation are significantly higher [1]. As the inventory continues to age, routine inspection practices will not be sufficient for the timely identification of areas of concern and to provide enough information to bridge owners to make informed decisions for safety and maintenance prioritization. Continuous monitoring is therefore desirable for long-term evaluation; monitoring areas of concern, such as retrofits or previous repairs or monitoring an area with known flaws, before a scheduled inspection. Continuous monitoring can also be used in cases where there is a concern about vandalism, terrorism, and/or bridge element integrity. Therefore, monitoring of fatigue cracking in steel bridges is of interest to many bridge owners and agencies.

To address this urgent need, the authors are conducting research on novel and promising sensing approaches together with energy harvesting devices to reduce the dramatic uncertainty inherent into steel bridge inspection and maintenance plan [2]. One of the challenges in this research is focused on the development of dual use piezoelectric wafer active sensors for fatigue crack detection. The combined schematic uses acoustic emission to detect the presence of fatigue cracks in their early stage while active sensing allows for the imaging and quantification of cracks using minimum number of sensors.

1.1. Crack Detection on Steel Bridge Structures. The monitoring of fatigue cracking in bridges has been approached with acoustic emission using either resonant or broadband 


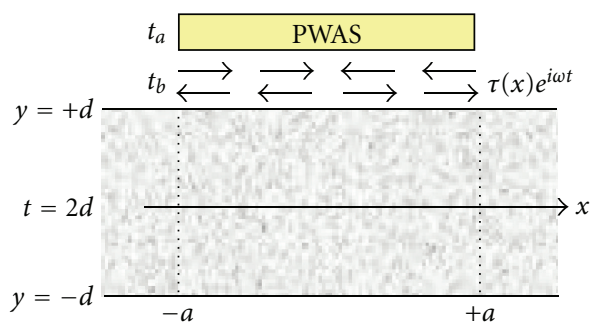

FIGURE 1: PWAS and structure interaction through the interface layer.

sensors. Acoustic emission monitoring is capable of detecting crack growth behavior [3-7] and assessing the integrity of structures such as bridges and aircraft $[8,9]$. Acoustic emission data has recently been directly related to crack growth rate in representative steel compact tension (CT) specimens. This development holds promise for the prognosis of inservice bridges [10]. The method has the notable advantage that the precise location of cracking does not need to be known for evaluation purposes. Rather, the sensors together with appropriate algorithms are capable of locating and quantifying active crack activity. It has been reported that acoustic emission techniques are so sensitive that fatigue cracks can be detected successfully even though the crack length may be less than $10 \mu \mathrm{m}[5,7]$. However, one of the challenges in passive monitoring is that the acoustic emission relies on an active crack growing process. Also, though acoustic emission sensing can detect a crack at its very early stage, it generally cannot provide information about the crack size or crack growth rate unless an initial size is provided.

Historically, AE signals have been captured with specially designed and fabricated AE sensors. Conventional AE sensors are made of piezoelectric crystals as the sensing elements which are encapsulated for protection and coupled together with a wear plate for good acoustic coupling. The frequency content and sensitivity of the sensor are controlled by the geometry and properties of the piezoelectric crystal as well as the housing for the crystal.

1.2. Piezoelectric Wafer Active Sensors. Piezoelectric wafer active sensors (PWAS) can function as an active sensing or passive device or network using piezoelectric principles and provide a correlation between mechanical and electrical variables. They can be permanently attached to the structure to monitor condition at will and can operate in active guided wave interrogation or passive AE sensing modes. The transmission of actuation and sensing between the PWAS and the host structure is achieved through the bonding adhesive layer. The adhesive layer (Figure 1) acts as a shear layer, in which the mechanical effects are transmitted through shear effects [11].

An important characteristic of PWAS, which distinguishes them from conventional ultrasonic transducers, is their capability of exciting multiple guided Lamb wave modes at a single frequency. There are at least two Lamb modes, A0 and S0, existing simultaneously when the product of the wave frequency and structure thickness $(f \cdot d$ product) falls in the range of $0 \sim 1 \mathrm{MHz}-\mathrm{mm}$. At larger $f \cdot d$ product values, more modes are present. In addition, due to the intrinsic dispersion property, the Lamb wave modes propagate at different speeds and the speeds change with frequency, which complicates the signal interpretation for damage detection. A single mode that is sensitive to the damage is desired for most of the SHM applications. This may be attained through wave tuning [12]. The process of wave tuning attempts to modify the excitation parameters to excite a certain mode for detection of a specified type or instance of damage [12]. By carefully selecting PWAS length at either an odd or even multiple of the half wavelength, a complex pattern of strain maxima and minima emerges (Figure 2). Since several Lamb modes, each with its own different wavelength, coexist at the same time, a selected Lamb mode can be tuned by choosing the appropriate frequency and PWAS dimensions.

An example of PWAS tuning is presented in Figure 2 for a $7-\mathrm{mm}$ square PWAS installed on a $1-\mathrm{mm}$ aluminum alloy 2024-T3 plate. The experimental amplitude plot in Figure 2(a) shows that for the plate being studied, a S0 tuning frequency around $200 \mathrm{kHz}$ can be identified, where the amplitude of the A0 mode is minimized while that of the S0 mode is still strong. Therefore, by choosing the excitation frequency, a single mode can be obtained for damage detection [13]. Theoretical prediction given in Figure 2(b) is consistent with the experimental results.

1.3. PWAS Dual Mode Sensing toward Field Application. Dupont et al. [14] have demonstrated the possibility of using embedded piezoelectric thin wafers to detect $\mathrm{AE}$ signals in composite materials. In the subject project, we are developing a dual mode sensing approach using the lowprofile wireless PWAS network with energy harvested from wind and/or ambient vibration energy [15]. To minimize the energy consumption, on one hand, it is envisioned that as few as four PWAS will be employed to monitor the crack growth. On the other hand, the dual mode sensing allows PWAS to operate in passive AE mode throughout the entire monitoring process unless significant AE events are detected, indicating major crack presence in the structure. When an AE event is identified, the PWAS can be switched to active mode to interrogate the bridge structure with propagating guided waves to assess the crack size and location. Eventually, an entire steel bridge may be mapped with the guided wave interrogation with visualized crack damage.

\section{PWAS Passive Mode Acoustic Emission Detection}

In the subject project, we adapted PWAS as AE sensors to detect stress waves with frequency components concentrated at $150 \mathrm{kHz}$ where the acoustic signals propagating with minimal attenuation and background noise due to the rubbing of structural components.

2.1. PWAS as AE Sensor. Laboratory tests have been conducted to investigate the PWAS application as an AE sensor. 

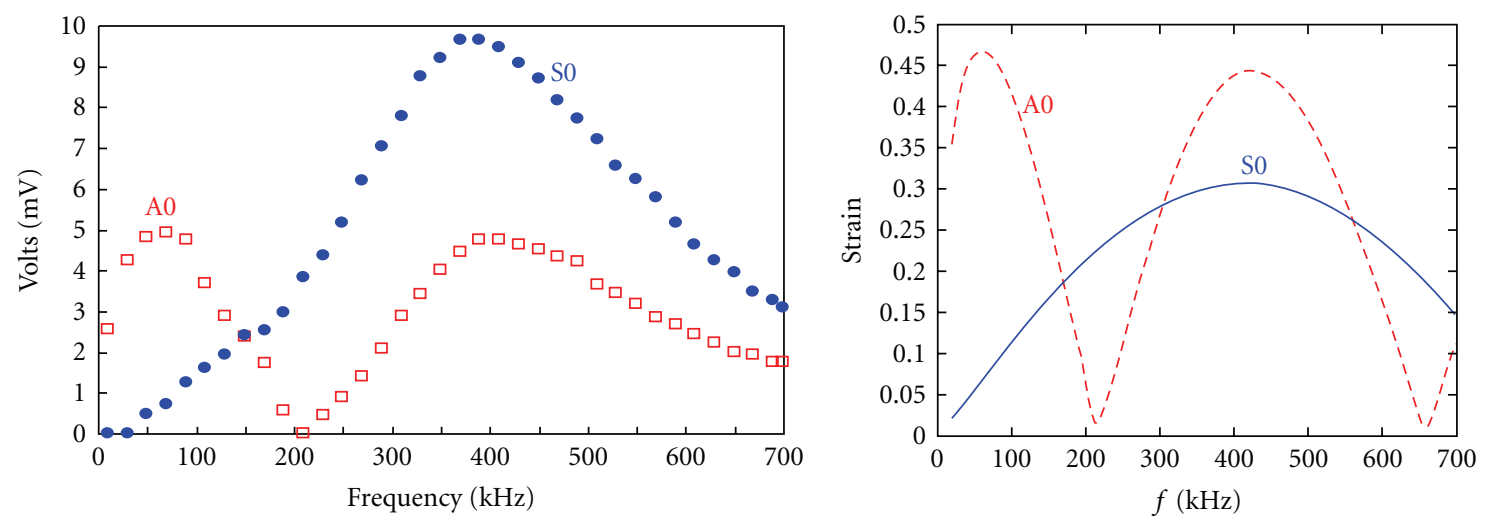

$\square \mathrm{A} 0$

- S0

(a)

(b)

FIGURE 2: Lamb wave mode tuning on a 1-mm thick aluminum alloy 2024-T3 using 7-mm PWAS. (a) Experimental wave amplitude within $0 \sim 700 \mathrm{kHz}$; (b) predicted strain curves [13].

A typical commercial R15I (http://www.pacndt.com/downloads/Sensors/Integral\%20Preamp/R15I-AST.pdf) AE sensor was used to calibrate the measurements. Two specimens were used in the tests. One was a $1.6 \mathrm{~mm}$ thick 2024 aluminum plate and the other was a $19 \mathrm{~mm}$ thick A572 grade 50 structural steel panel. Both specimens were installed with 7 -mm diameter $0.2 \mathrm{~mm}$ thick round PWAS Material APC 850 by Americanpiezo (http://www.americanpiezo.com/apcmaterials/choosing.html) using M-200 bonding adhesive following the standard installation used by strain gauge. The R15I sensor was mounted using hot glue.

The test setup is illustrated in Figure 3. PAC DiSP (http:// www.pacndt.com/index.aspx?go=products\&focus $=/$ multichannel//disp.htm) system was used to perform data acquisition. PWAS was connected to a preamp then to channel 1. The preamp had a $100-1200 \mathrm{kHz}$ built-in filter and could accommodate a signal amplification of $40 \mathrm{~dB}$. The R15I sensor had a built-in internal preamp with a gain also at $40 \mathrm{~dB}$ and was connected to channel 2. AE events were introduced by pencil lead break (PLB). Rubber gloves were used to avoid causing electrical disturbance by touching the plate.

2.1.1. Aluminum Plate Tests. In the first part of the work, a $1.6-\mathrm{mm}$ thick aluminum plate, approximately $300-\mathrm{mm}$ by 300-mm, was used for testing PWAS AE detection. PWAS and R15I were placed adjacently on the plate, about $165 \mathrm{~mm}$ away from the plate edge where the PLB was applied. In total, five PLB of various lead sizes were applied. The PWAS transducer detected all of them with comparable amplitudes to those captured by R15I, as summarized in Table 1 .

Figure 4 shows the waveforms and the frequency spectra for the test described in the third row in Table 1. In this test, $0.5 \mathrm{~mm} \mathrm{HB}$ pencil lead was broken at the edge of the plate in the out-of-plane direction (pressing down and springing up). The detected AE signal amplitudes of the two sensors were about the same $(78 \mathrm{~dB})$. The PWAS gave a peak signal of
TABle 1: AE detection on the 1.6-mm aluminum plate.

\begin{tabular}{lcc}
\hline PLB size & PWAS AE amplitude $(\mathrm{dB})$ & R15I AE amplitude $(\mathrm{dB})$ \\
\hline $0.7 \mathrm{~mm}$ & 83 & 82 \\
$0.5 \mathrm{~mm}$ & 89 & 89 \\
$0.5 \mathrm{~mm}$ & 78 & 78 \\
$0.3 \mathrm{~mm}$ & 88 & 86 \\
$0.3 \mathrm{~mm}$ & 84 & 78 \\
\hline
\end{tabular}

$2200 \mathrm{mV}$ against a noise of $200 \mathrm{mV}$, resulting in a signal-tonoise ratio (SNR) of approximately 11 or 21 in $\mathrm{dB}$. Compared to R15I waveform, the response of PWAS was conspicuously crisper in the earlier part corresponding to the S0 mode arrival which was followed by the arrival of slower A0 mode.

Looking at the AE waveforms by PWAS and R15I present in Figure 4, it is noticed though the two waveforms have comparable signal peak amplitudes, PWAS shows higher floor noise (circled part). For AE detection, it is needed that the sensor shows good signal-to-noise ratio. In this case, floor noise for PWAS is too high and needs to be decreased.

An expanded view of the early PWAS response is shown in Figure 5(a). The fast S0 mode and slow but highly dispersive A0 mode are clearly present. The out-of-plane displacement waveform generated by PlotRLQ (PlotRLQ is a computer program that computes theoretical waveforms by summing the contributions of Lamb wave modes excited by specified moment tensor sources at specified depths within the plate based on classic Lamb wave theory) calculated for this situation is shown in Figure 5(b). The agreement between PWAS waveform and theoretical prediction is discernable, especially for the S0 mode, the earliest part of the response. The small discrepancy comes from the ragged appearance at the beginning of the A0 mode and from the relatively abrupt falloff after the A0 reaches its peak amplitude. This may be credited to the PWAS sensor aperture effect as described in [12]. 


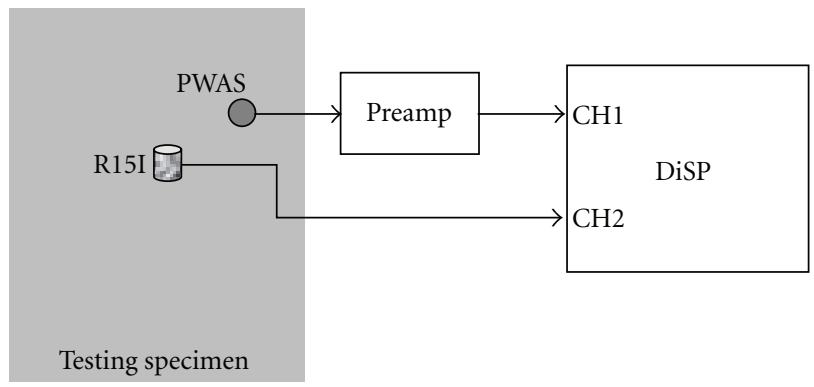

(a)

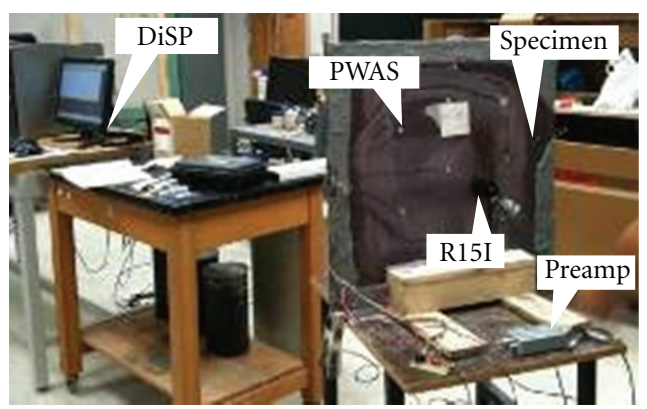

(b)

Figure 3: AE testing setup. (a) Test setup schematic; (b) laboratory test setup.
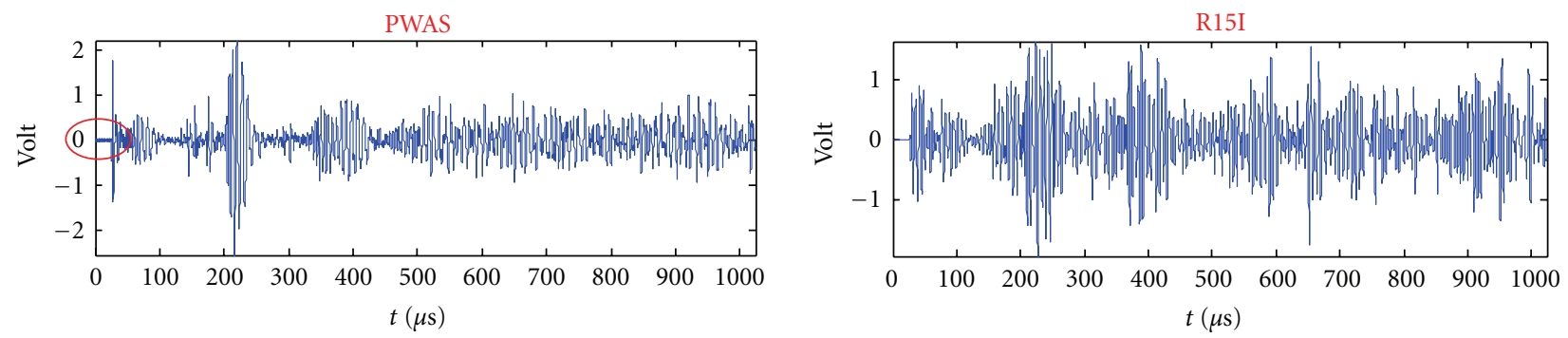

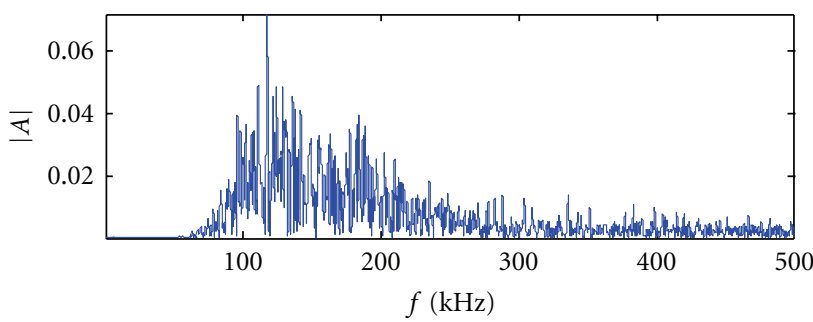

(a)

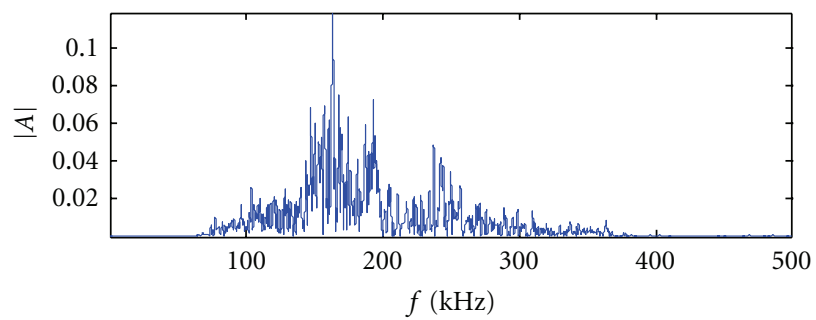

(b)

FIGURE 4: $0.5 \mathrm{~mm}$ PLB detection on 1.6- $\mathrm{mm}$ aluminum plate. (a) PWAS AE waveform and its frequency spectrum; (a) R15I AE waveform and its frequency spectrum.

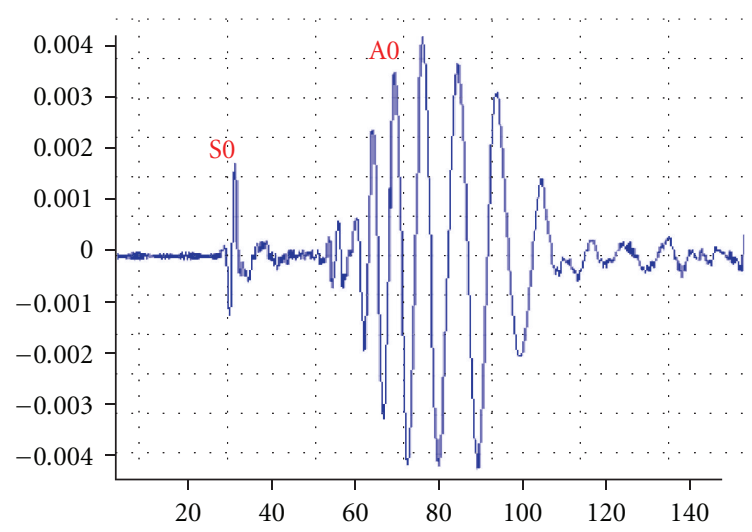

(a)

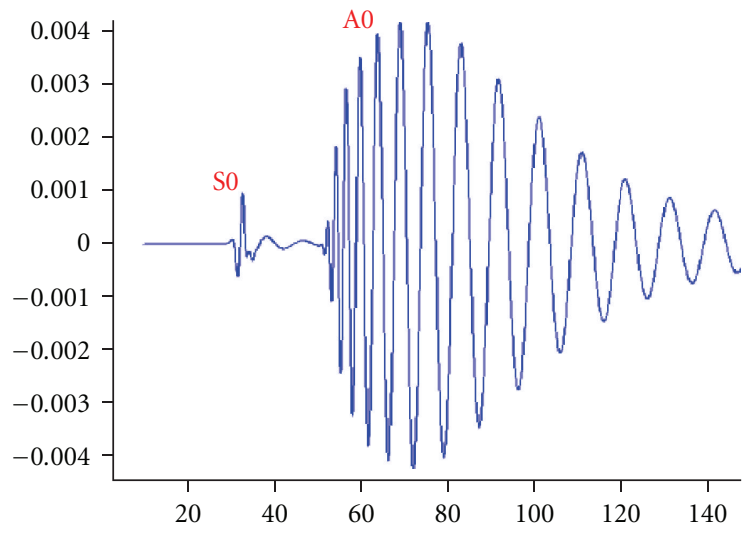

(b)

FIGURE 5: PWAS response compared to theoretical prediction. (a) PWAS waveform; (b) theoretical out-of-plane displacement by PlotRLQ. 

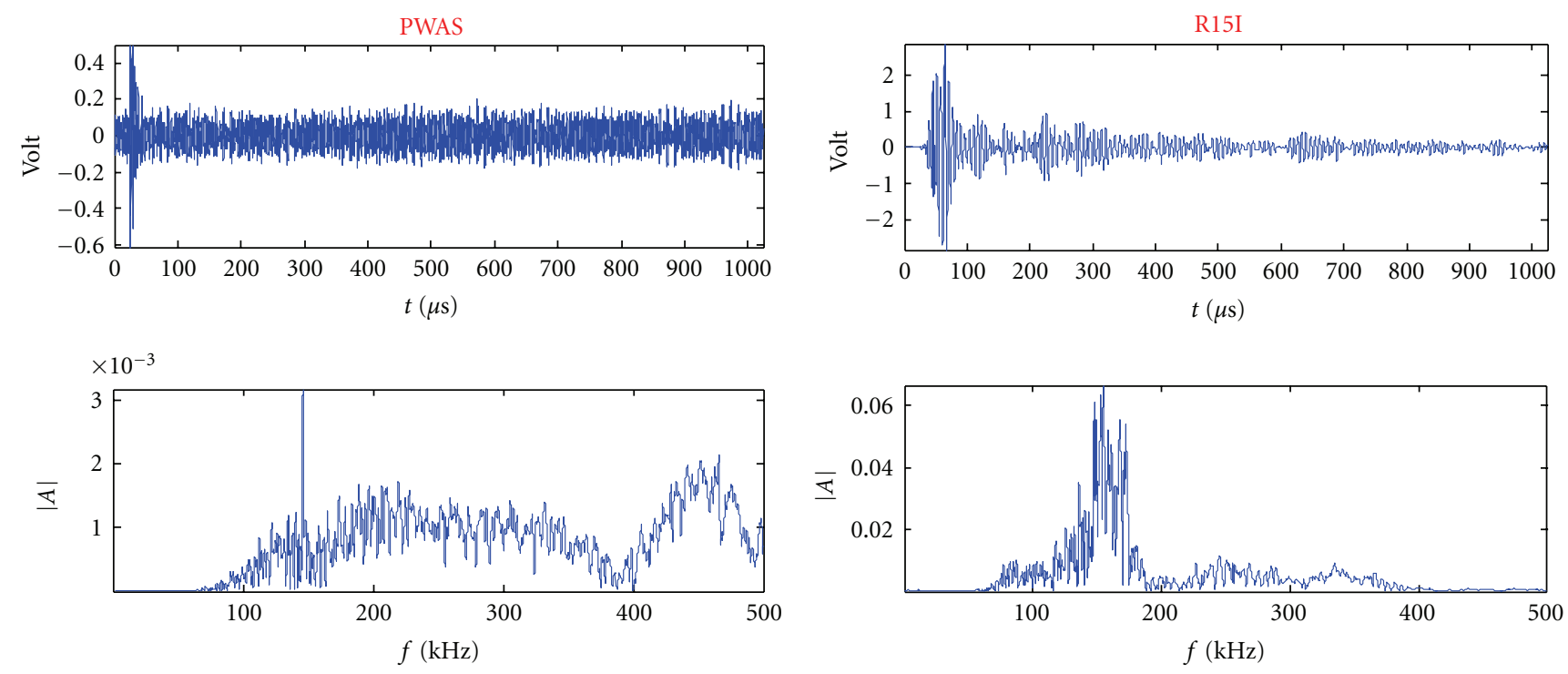

(a)

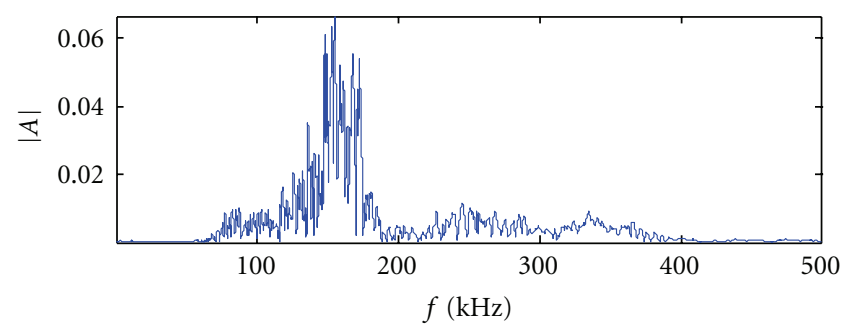

(b)

FIGURE 6: $0.5 \mathrm{~mm}$ PLB detection on 19-mm steel plate. (a) PWAS AE waveform and its frequency spectrum; (a) R15I AE waveform and its frequency spectrum.

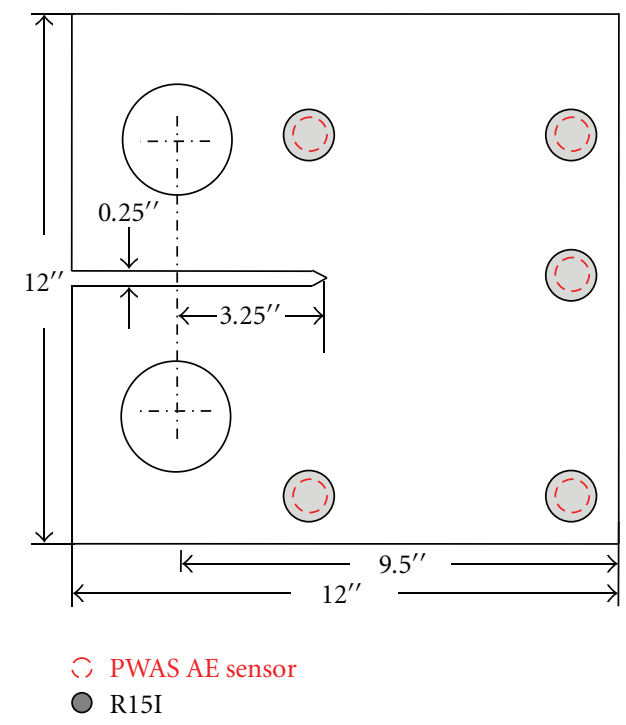

(a)

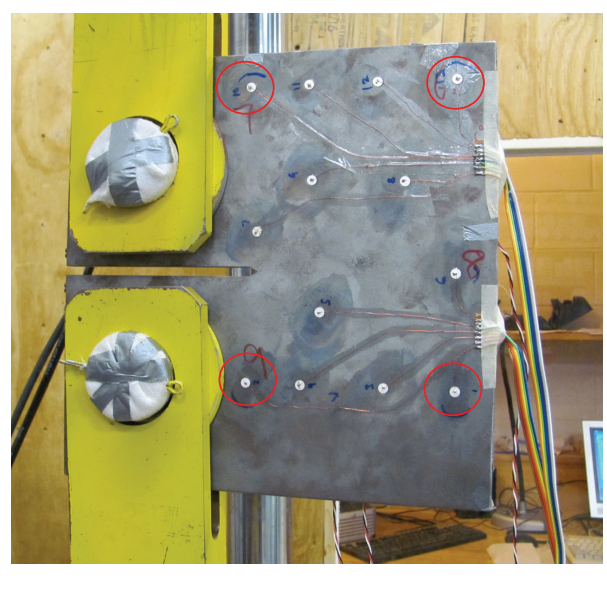

(b)

FIgURE 7: AE detection on a 1/2" CT specimen. (a) Geometry of the specimen and arrangement of transducers; (b) a snapshot of the actual specimen. AE PWAS are circled (rest are for active sensing). The R15I were installed on the other side of the specimen.

2.1.2. Steel Plate Tests. The second part of the work was conducted on a 19-mm thick steel plate. $0.5 \mathrm{~mm}$ HB PLB was applied on the surface of the plate about $72 \mathrm{~mm}$ away from PWAS. The R15I transducer was glued on the plate with a distance of $98 \mathrm{~mm}$ from the PLB.

The PLB was detected by PWAS with an AE amplitude of $73 \mathrm{~dB}$ in contrast to the $87 \mathrm{~dB}$ detected by R15I. The waveforms and their frequency spectra are provided in Figure 6. The PWAS signal showed a strong negative-going spike accounting for the $73 \mathrm{~dB}$ amplitude, fitting in a $600 \mathrm{mV}$ peak. The background noise was about $150 \mathrm{mV}$, resulting in a
SNR of approximately 4 or about 12 in $\mathrm{dB}$. The SNR of PWAS in steel plate was much lower than that in aluminum plate.

By examining the frequency spectra, it can be noted that PWAS has major frequency components beyond $200 \mathrm{kHz}$, showing a wider frequency response compared to resonant type R15I AE sensor.

\subsection{PWAS AE Detection in Compact Tension Testing. Com-} pact tension (CT) specimens made of the same material as the steel plate used in Section 2.1 were used. The geometry of the specimens is displayed in Figure 7. Custom fixtures 


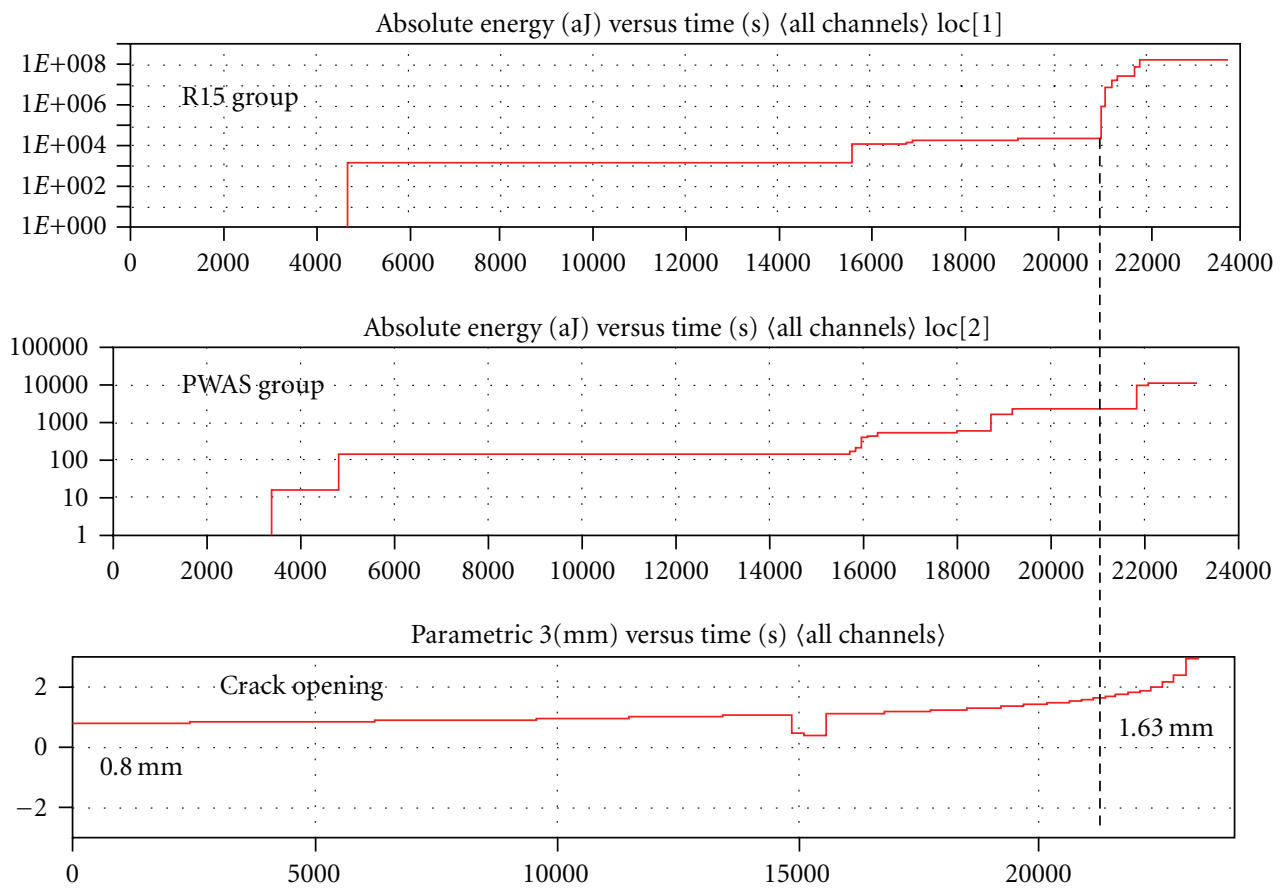

(a)

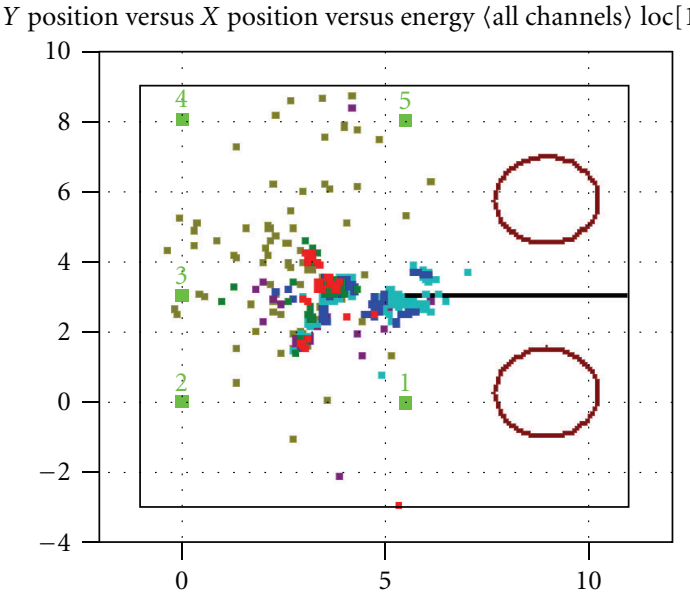

(b)

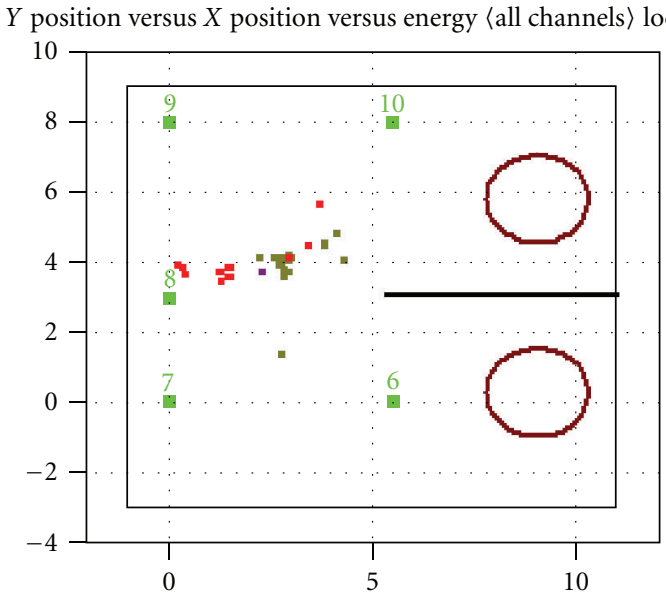

(c)

FIGURE 8: Comparison of crack localization in CT test on $1 / 2^{\prime \prime}$ steel specimen. (a) Cumulative acoustic energy by PWAS and R15I; (b) cracking detection and localization by R15I; (c) cracking detection and localization by PWAS.

were designed and fabricated to mount the CT specimens. The cyclic tension loads of minimum $1 \mathrm{KN}$ and maximum $50 \mathrm{KN}$ were applied to the specimen using an MTS810 servohydraulic mechanical testing machine. Fatigue tests were conducted under load-controlled mode with frequency of $1 \mathrm{HZ}$. A clip gage was employed to measure the crack mouth opening displacement (CMOD) to clarify crack opening and closure and to determine the magnitude of the CMOD. The surface cracks were also monitored optically with a high-resolution recording microscope. Two separate sets of AE sensors, namely, R15I and PWAS monitored the process, as well. They connected to the Sensor Highway II
(http://www.pacndt.com/products/Remote\%20Monitoring/ AE_Sensor_Highway.pdf) data acquisition system through preamps. The data from these two sets of sensors were analyzed separately with AEwin (http://www.pacndt.com/index .aspx?go=products\&focus=/software/aewin.htm) software.

The results of crack localization from PWAS sensors and R15I during CT testing are shown in Figure 8. R15I sensors detected 1,171 AE events prior to failure while PWAS detected only 54 events. Figure 8(a) gives the cumulative acoustic energy of R15I and PWAS together with the crack opening displacement. While PWAS detected a fewer number of acoustic activities, they detected the crack growth when 


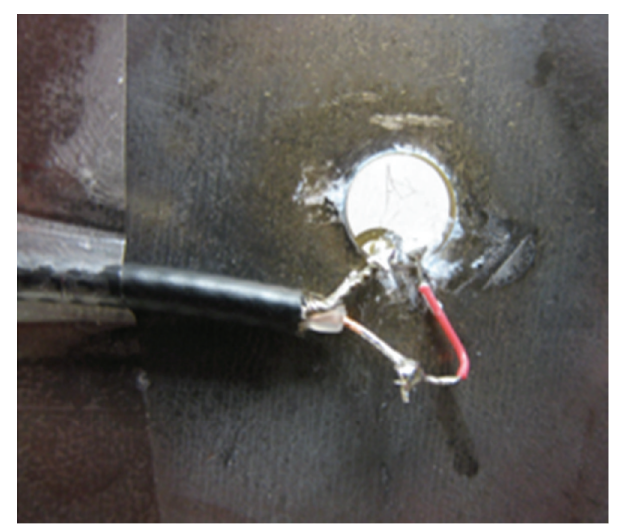

Figure 9: PWAS installation using a coaxial cable.
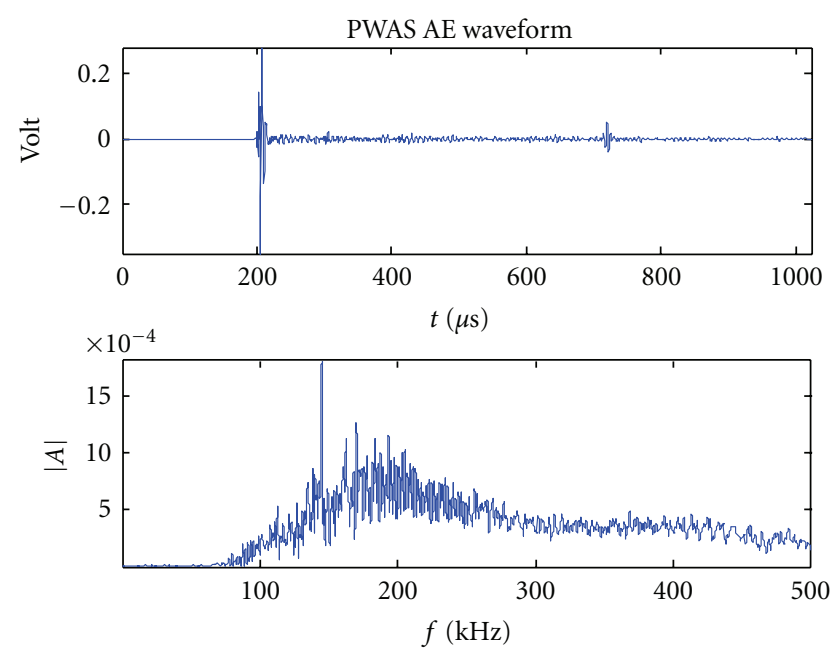

Figure 10: PLB detection on steel plate using coaxial cable wired PWAS.

the crack size reaches $0.83 \mathrm{~mm}$. As can be seen in Figure 8(b), PWAS localization was closer and concentrated around the crack tip compared with the R15I detection in Figure $8(\mathrm{c})$. This was thought to be mainly due to the enhanced sensitivity of the R15I sensors, which can complicate source location in small-scale laboratory specimens due to reflections.

2.3. PWAS Adaptation toward Field Application. In the validation tests, it has been noticed that PWAS on steel specimens exhibited higher floor noise compared to the standard R15I AE sensors, therefore, providing a poor SNR ratio. To enhance the signal quality toward field application and decrease the background floor noise, we improved PWAS installation by using a coaxial cable similar to that used for R15I sensors. The shield of the coax was connected to the steel plate very close to the PWAS while the center conductor was connected to the positive electrode of the PWAS, as shown in Figure 9.

Detection of a $0.3 \mathrm{~mm}$ PLB about $20 \mathrm{~mm}$ away from a coaxial cable wired PWAS was evaluated (Figure 10). The rest of the setup remained the same as for the tests presented in Section 2.1.2. The resulting waveform had an amplitude of $71 \mathrm{~dB}$, approximately $400 \mathrm{mV}$. The background floor noise was discernibly decreased and approximated at $10 \mathrm{mV}$. Therefore, SNR was measured at 40 or $32 \mathrm{~dB}$, significantly improved compared to the $12 \mathrm{~dB}$ presented in Section 2.1.2.

\section{PWAS Active Mode Crack Sensing}

In the dual mode sensing schematic, after significant cracking has been identified by passive mode $\mathrm{AE}$ detection, active mode sensing using pitch-catch interrogation is evoked to quantify crack growth through damage index and array imaging. A PWAS network consisting of several sensors spatially distributed on the plate can be used to interrogate the plate with one sensor generating the guided wave and the others receiving the structural response. When an elastic Lamb wave is transmitted and travels through the structure, wave scattering occurs in all directions where there is a change in the material properties due to damage. The scatter signal is defined as the difference between the measurement during the development of damage and the baseline signal at the initial stage. One advantage of using scatter signals is to minimize the influence caused by boundaries or other structural features which would otherwise complicate the Lamb wave analysis.

3.1. Damage Index Evaluation. We assume that cracking is the sole source of changes in the detected Lamb waves. Also being assumed is that the waves travel in straight paths in the plate structures. Hence, the objective of our Lamb wave signal analysis is to extract damage-related characteristics from the measured sensory data. In this research damage index (DI) is defined as [16]

$$
\mathrm{DI}=1-\frac{C_{X Y}}{\sigma_{X} \sigma_{Y}}
$$

$C_{X Y}$ is the covariance of $X$ and $Y$ given by

$$
C_{X Y}=\sum_{j=1}^{N}\left(X_{j}-\mu_{X}\right)\left(Y_{j}-\mu_{Y}\right) .
$$

where $\mu$ is the mean value and $N$ is the length of the dataset. $\sigma_{X}$ and $\sigma_{Y}$ are the standard deviations of $X$ and $Y$, respectively, with their product given as

$$
\sigma_{X} \sigma_{Y}=\sqrt{\sum_{j=1}^{N}\left(X_{j}-\mu_{X}\right)^{2}\left(Y_{j}-\mu_{Y}\right)^{2}} .
$$

For the active sensing implemented during the CT test presented in Section 2.2, four PWAS were used to perform pitch-catch wave propagation interrogation, as marked and numbered in Figure 11(a). Using the definition of damage index defined above, the DI curves were plotted at different crack length as shown in Figure 11(b) for all the pitch-catch paths. The DI increases when the crack grows. The detection along sensors P0 to P1 is most sensitive, followed by the one 

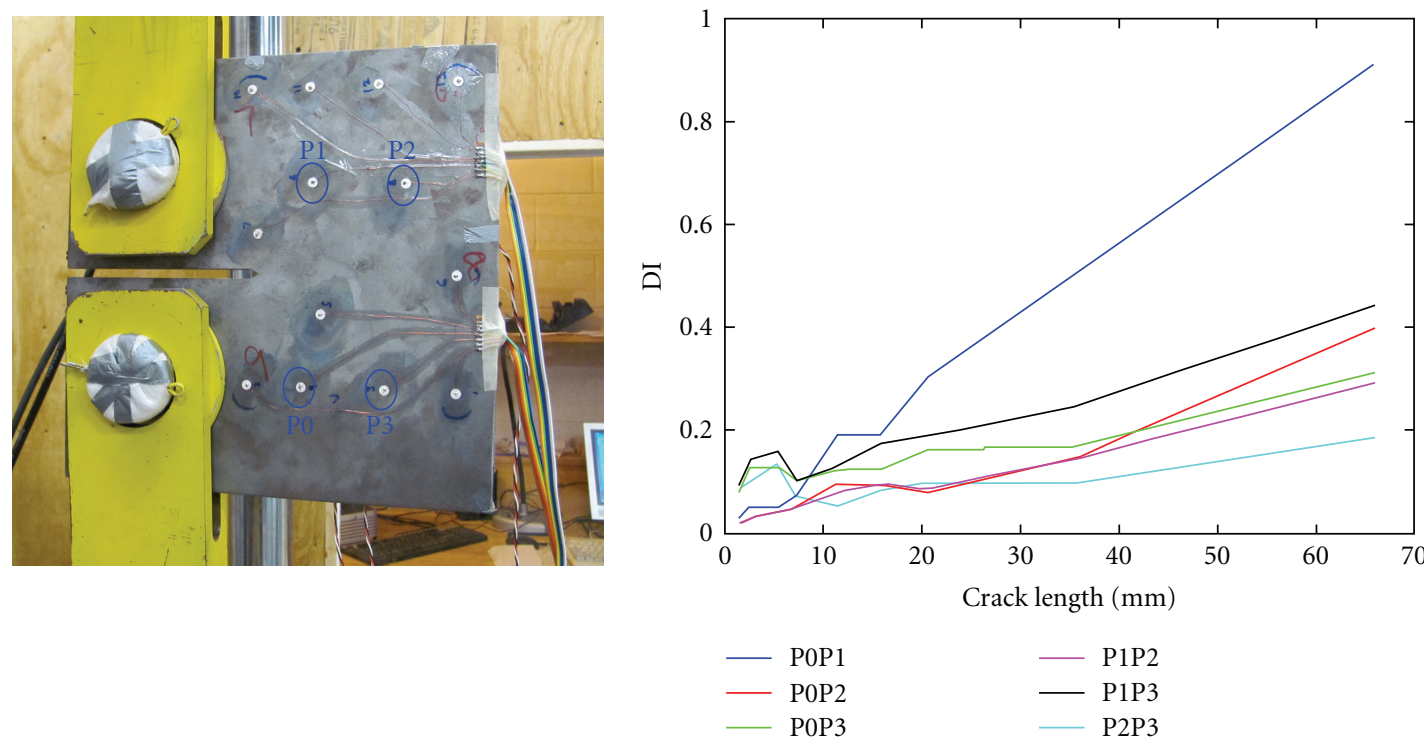

(a)

(b)

FIGURE 11: AE detection on a 1/2" CT specimen. (a) Geometry of the specimen and arrangement of transducers; (b) a snapshot of the actual specimen. AE PWAS are circled (rest are for active sensing). The R15I was installed on the other side of the specimen.

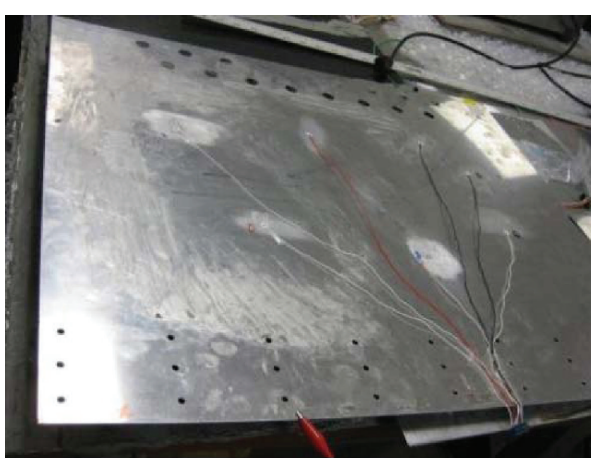

(a)

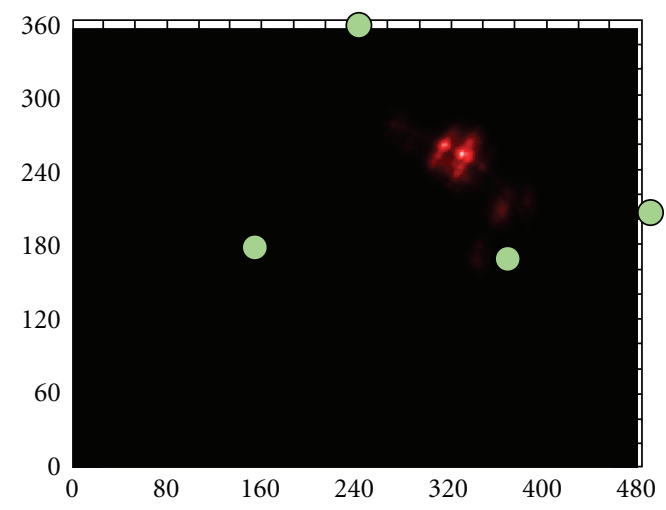

(b)

FIGURE 12: Array imaging for crack growth detection. (a) Test specimen and sensor network; (b) array imaging of a hairline crack of 18 mm length. Sensor locations are marked as green face circles.

along sensors P1 to P3 since the paths are perpendicular to the crack development path. The increment of the DI curves is also well correlated to the crack growth under fatigue loading.

3.2. Array Imaging. The array imaging methods have the incredible capability to map the structure and existing damage in it, providing a means to qualitatively assess the structural integrity. The sparse array uses scatter signals from a network of sensors to construct a diagnosis image. The image construction is based on triangulation principle and conducted by shifting back the scatter signals at time quantities defined by the transmitter-receiver locations used in the pitch-catch mode. Assuming a single damage scatter is located at point $Z(x, y)$ in the structure, the scatter signal from transmitter $T_{i}$ to receiver $R_{j}$ contains a single wave packet caused by the damage. The total time of traveling $\tau_{Z}$ is determined by the locations of the transmitter $T_{i}$ at $\left(x_{i}, y_{i}\right)$, the receiver $R_{j}$ at $\left(x_{j}, y_{j}\right)$, and $Z(x, y)$, as

$$
\tau_{Z}=\frac{\sqrt{\left(x_{i}-x\right)^{2}+\left(y_{i}-y\right)^{2}}+\sqrt{\left(x-x_{j}\right)^{2}+\left(y-y_{j}\right)^{2}}}{c_{g}},
$$

where $c_{g}$ is the group velocity of the traveling Lamb wave, assuming constant. When a wave packet is shifted back by the quantity defined by the transducers and the exact position of the damage, that is, $\tau_{Z}$, ideally the peak will be shifted right back to the time origin. If the wave packet is shifted by a quantity defined with otherwise cases (such as $\tau_{i}$ and 
$\left.\tau_{O}\right)$, the peak will not be shifted right at the time origin. For an unknown damage, for a certain scatter signal with $\tau_{Z}$, the possible locations of the damage constitute an orbit of ellipse with the transmitter and receiver as the foci. To locate the damage, ellipses from other scatters (or transmitter-receiver pairs in the network) are needed (triangulation). For a given network of $M$ transducers, a total of $M^{2}$ scatter signals will be used without considering reciprocity. The pixel value at the location $Z(x, y)$ is defined as

$$
P_{Z}\left(t_{0}\right)=\prod_{i=1}^{M} \prod_{j=1}^{M} s_{i j}\left(\tau_{Z}\right), \quad i \neq j,
$$

where $s_{i j}$ is the scatter signal obtained from $i$ th transmitter and $j$ th receiver. Details of principles and applications of ultrasonic array imaging can be found in [17].

The crack detection demonstration was conducted on a $1-\mathrm{mm}$ thick aluminum plate. The imaging was performed by a four-PWAS network to assess a simulated hairline crack centered inside, shown in Figure 12(a). A hairline crack of $18 \mathrm{~mm}$ was made on the plate, and then crack length was increased to $23 \mathrm{~mm}$, resulting in a growth of $5 \mathrm{~mm}$. The Lamb wave used to conduct the imaging was the S0 mode at $310 \mathrm{kHz}$ with a wavelength about $17 \mathrm{~mm}$.

The image result of the crack at $18 \mathrm{~mm}$ is shown in Figure 12(b), giving a clear detection with two highlighted spots representing the two crack tips; therefore, they could be used to estimate the crack length and monitor the crack growth. The first crack of $18 \mathrm{~mm}$ was estimated at $17.09 \mathrm{~mm}$ and the second one of $23 \mathrm{~mm}$ was estimated at $22.47 \mathrm{~mm}$. A crack growth of $5.38 \mathrm{~mm}$ was hence measured with the array imaging method with an error of about $7.6 \%$ compared to the actual growth of $5 \mathrm{~mm}$ (from $18 \mathrm{~mm}$ to $23 \mathrm{~mm}$ ).

\section{Conclusions}

Piezoelectric wafer active sensors (PWAS) have made tremendous progress in structural health monitoring during the past decade, but their exposure to civil infrastructure has been little discussed so far. The work presented here intends to explore PWAS applications for in-field monitoring of infrastructure (e.g., civil steel bridges) using both acoustic emission and active wave propagation sensing. Laboratory demonstration on both thin aluminum and thick steel plates, the PWAS have been proved as AE sensors. The use of coaxial wiring cables has greatly improved the PWAS waveform signal-to-noise ratio, making it more suitable for field application. The PWAS AE sensing of fatigue cracking on a CT specimen showed that it can provide concentrated detection around the crack tip with a relatively fewer numbers of acoustic emission events than the R15I AE transducers. The PWAS active mode sensing using propagating guided waves can interrogate the structures at will and provide a clear indication and quantitative estimation of the crack growth through damage index or array imaging. The dual mode sensing of the presented PWAS methodology has shown its promising application to insitu health monitoring and diagnosis of steel bridges.

\section{Acknowledgments}

This paper was performed under the support of the US Department of Commerce, National Institute of Standards and Technology, Technology Innovation Program, Cooperative Agreement number 70NANB9H9007. The authors would also like to thank Dr. Adrian Pollock from Mistras Group for his insightful comments on the presented passive sensing.

\section{References}

[1] I. M. Friedland, H. Ghasemi, and S. B. Chase, The FHWA Long-Term Bridge Performance Program, Federal Highway Administration, Turner-Fairbank Highway Research Center, McLean, Va, USA, 2007.

[2] L. Yu, V. Giurgiutiu, P. Ziehl, P. Pollock, and D. Ozevin, "Steel bridge fatigue crack detection with piezoelectric wafer active sensors," in Sensors and Smart Structures Technologies for Civil, Mechanical, and Aerospace Systems, vol. 7647 of Proceedings of SPIE, San Diego, Calif, USA, March 2010.

[3] A. C. E. Sinclair, D. C. Connors, and C. L. Formby, "Acoustic emission analysis during fatigue crack growth in steel," Materials Science and Engineering, vol. 28, no. 2, pp. 263-273, 1977.

[4] R. I. Stephens, S. G. Lee, and H. W. Lee, "Constant and variable amplitude fatigue behavior and fracture of A572 steel at $25^{\circ} \mathrm{C}\left(77^{\circ} \mathrm{F}\right)$ and $-45^{\circ} \mathrm{C}\left(-50^{\circ} \mathrm{F}\right), "$ International Journal of Fracture, vol. 19, no. 2, pp. 83-98, 1982.

[5] M. N. Bassim, S. S. Lawrence, and C. D. Liu, "Detection of the onset of fatigue crack growth in rail steels using acoustic emission," Engineering Fracture Mechanics, vol. 47, no. 2, pp. 207-214, 1994.

[6] A. Berkovits and D. Fang, "Study of fatigue crack characteristics by acoustic emission," Engineering Fracture Mechanics, vol. 51, no. 3, pp. 401-416, 1995.

[7] D. H. Kohn, P. Ducheyne, and J. Awerbuch, "Acoustic emission during fatigue of Ti-6Al-4V: Incipient fatigue crack detection limits and generalized data analysis methodology," Journal of Materials Science, vol. 27, no. 12, pp. 3133-3142, 1992.

[8] Z. Gong, E. O. Nyborg, and G. Oommen, "Acoustic emission monitoring of steel railroad bridges," Materials Evaluation, vol. 50, no. 7, pp. 883-887, 1992.

[9] H. L. Chen and J. H. Choi, "Acoustic emission study of fatigue cracks in materials used for AVLB," Journal of Nondestructive Evaluation, vol. 23, no. 4, pp. 133-151, 2004.

[10] J. Yu, P. Ziehl, B. Zrate, and J. Caicedo, "Prediction of fatigue crack growth in steel bridge components using acoustic emission," Journal of Constructional Steel Research, vol. 67, no. 8, pp. 1254-1260, 2011.

[11] E. F. Crawley and J. de Luis, "Use of piezoelectric actuators as elements of intelligent structures," AIAA Journal, vol. 25, no. 10, pp. 1373-1385, 1987.

[12] V. Giurgiutiu, Structural Health Monitoring with Piezoelectric Wafer Active Sensors, Elsevier Academic Press, 2008.

[13] G. B. Santoni, L. Yu, B. Xu, and V. Giurgiutiu, "Lamb wavemode tuning of piezoelectric wafer active sensors for structural health monitoring," Journal of Vibration and Acoustics, vol. 129, no. 6, pp. 752-762, 2007.

[14] M. Dupont, R. Osmont, R. Gouyon, and D. L. Balageas, "Permanent monitoring of damaging impacts by a piezoelectric sensor based integrated system," in Proceedings of the 2nd International Workshop on Structural Health Monitoring, pp. 
561-570, Stanford University, Stanford, Calif, USA, September 2000.

[15] L. Yu, V. Giurgiutiu, P. Ziehl, and D. Ozevin, "Piezoelectric based sensing in wireless steel bridge health monitoring," in Nondestructive Characterization for Composite Materials, Aerospace Engineering, Civil Infrastructure, and Homeland Security, vol. 7294 of Proceedings of SPIE, San Diego, Calif, USA, March 2009.

[16] X. Zhao, H. Gao, G. Zhang et al., "Active health monitoring of an aircraft wing with embedded piezoelectric sensor/actuator network: I. Defect detection, localization and growth monitoring," Smart Materials and Structures, vol. 16, no. 4, pp. 12081217, 2007.

[17] L. Yu and V. Giurgiutiu, "Piezoelectric wafer active sensor guided wave imaging," in Smart Sensor Phenomena, Technology, Networks, and Systems, vol. 7648 of Proceedings of SPIE, San Diego, Calif, USA, March 2010. 

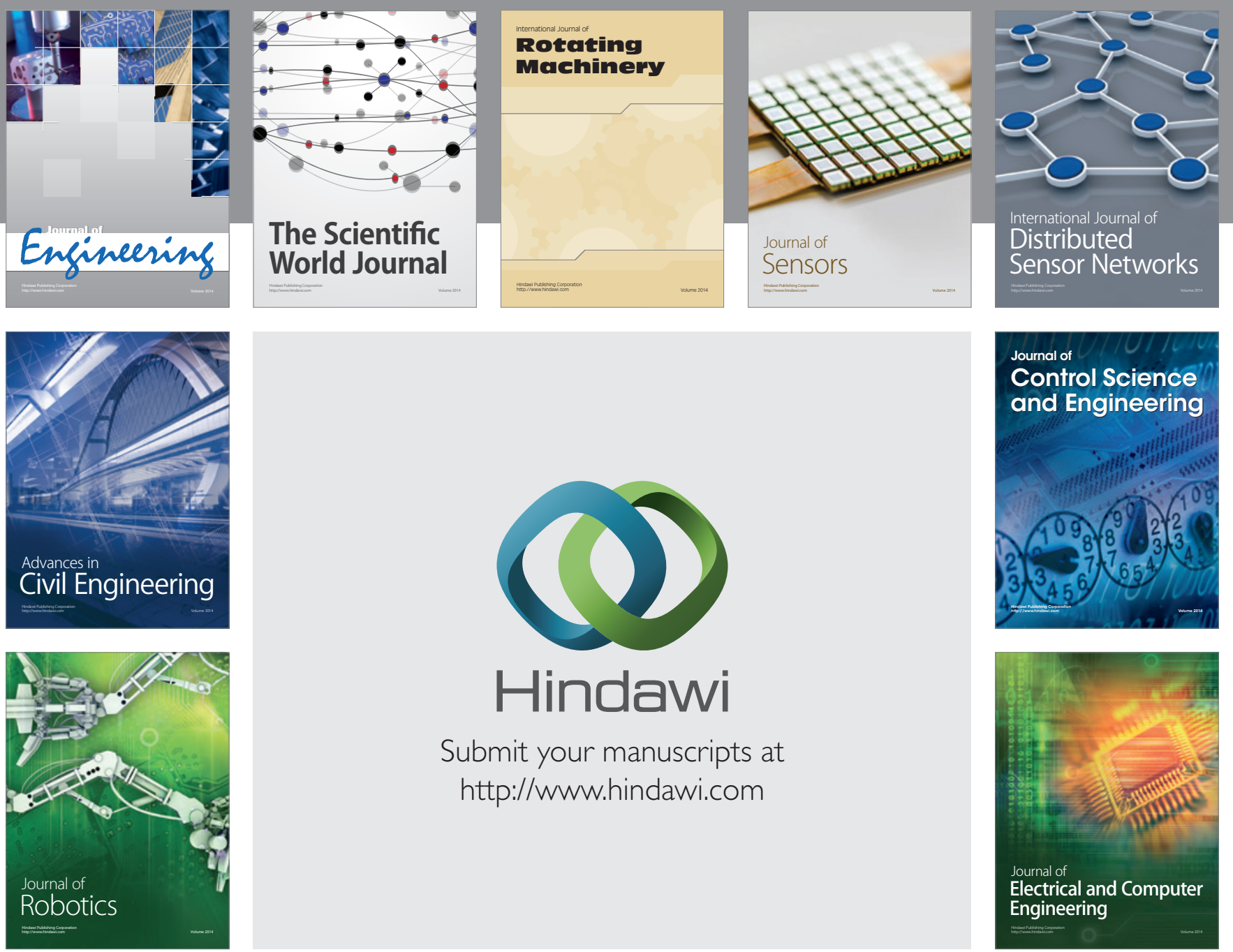

Submit your manuscripts at

http://www.hindawi.com
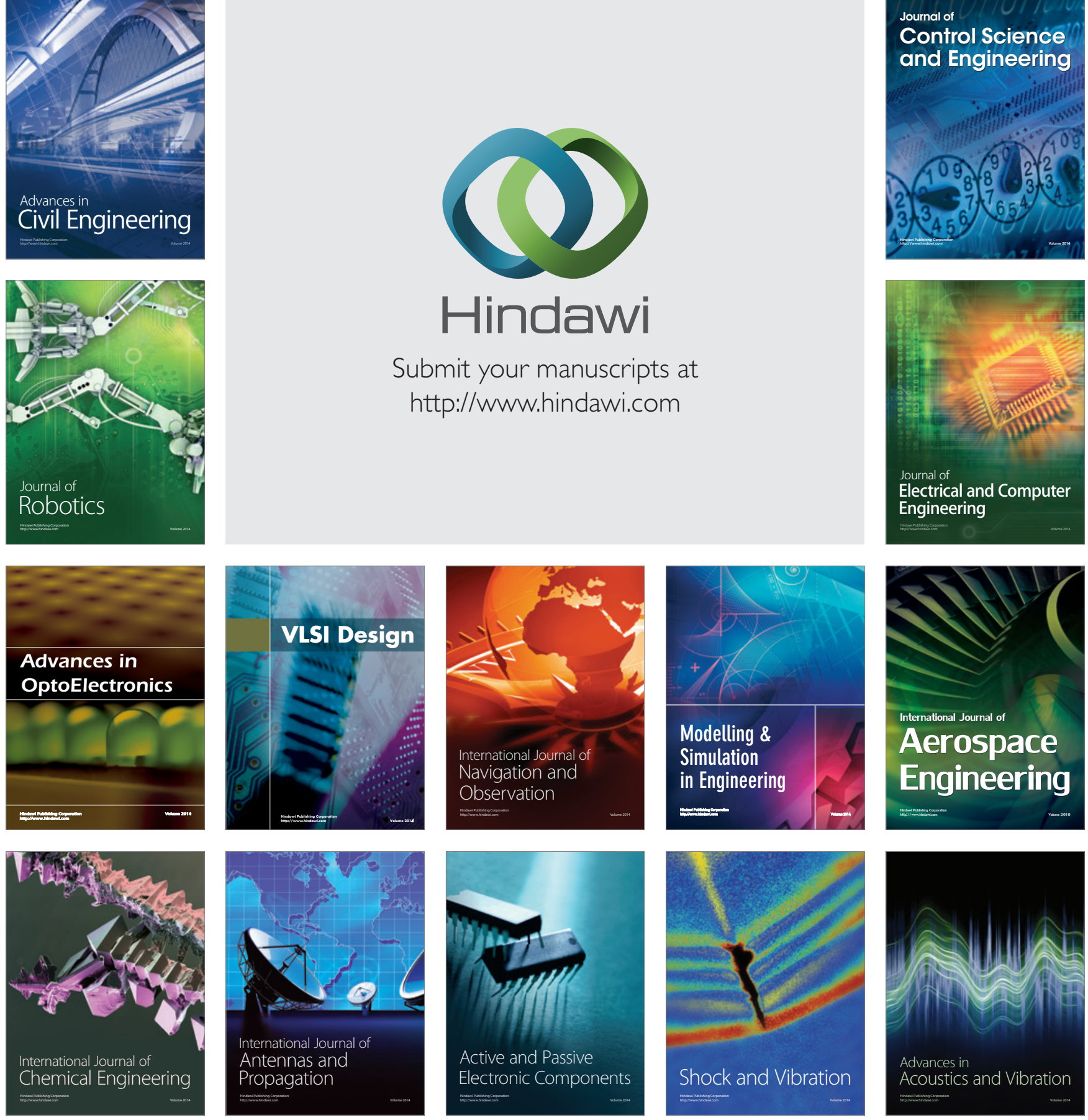